\title{
A New Kalman Filter Based State Estimation Method for Multi-input Multi-output Unit Time-Delay Systems
}

\author{
Behrouz Safarinejadian $^{1 *}$ and Mohiyeddin Mozaffari² \\ 'Department of Electrical Engineering, Shiraz University of Technology, safarinejad@sutech.ac.ir. \\ ${ }^{2}$ Department of Electrical Engineering, Shiraz University of Technology, m.mozaffari@sutech.ac.ir.
}

\begin{abstract}
A new state estimation method for unit time-delay systems will be presented in this paper. Kalman filter will be considered to estimate the states of a linear system with unit time-delay. First, an augmented state vector is defined which consists of the main state vector and the state vector with a unit time-delay. The states of the augmented system will be estimated using Kalman filter algorithm. From the augmented state vector, the main state vector can be obtained. Finally, an example is provided to test the performance of the proposed method.
\end{abstract}

Keywords: Kalman Filter, State Estimation, Augmented System, Time Delay.

\section{Introduction}

State space models have wide applications in many areas, e.g., system identification [1-3], adaptive filtering [4-6], system modelling and control [7, 8], energy and power systems $[9,10]$, signal processing $[11,12]$. Most contributions for systems with time-delay focus on the studies of control schemes. For example, Yan and Shi discussed the robust discrete-time sliding mode control for the uncertain systems with time-varying state delay [13], Shi and Yu studied the output feedback stabilization of the networked control systems with random delays modelled by Markov chains [14].

The Kalman filter has played a central role in system theory and has found wide applications in many fields such as control, signal processing, and communication [15]. There are various algorithms for state estimation such as Kalman filter, extended Kalman filter, unscented Kalman filter, optimal smoothing and particle filters. The basic idea in all of the mentioned methods is the Kalman filter. In this paper, due to linear dynamic of the system, Kalman filter will be used for state estimation.

This paper considers estimation problem of time-delay linear control systems based on the augmented system idea. The augmented system is a new-type state estimation and deals with estimation problems using augmented state vector. The basic idea is to define a new state vector including the main state vector and state vector with a unit time-delay. Then, by implementing Kalman filter algorithm for estimating the states, the main state vector can be obtained from the augmented state vector.

Briefly, this paper is organized as follows. In Section 2, an input-output representation related to the state space model with a unit time-delay is derived. Section 3 presents transfer function and input-output representation of augmented system. In Section 4, a new Kalman filter algorithm for the augmented system will be presented. In Section 5, an illustrative example will be provided to verify the performance of the proposed algorithm. Finally, concluding remarks are given in Section 6.

* Corresponding author:

Behrouz Safarinejadian (safarinejad@sutech.ac.ir) 


\section{Input-Output Representation of System with Unit Time-Delay}

Consider the following state space model with a unit time-delay [16],

$$
\begin{aligned}
x(k+1) & =A x(k)+B x(k-1)+g u(k) \\
y(k) & =h x(k)
\end{aligned}
$$

where $x(k) \in \mathbb{R}^{n}$ is the state vector, $u(k) \in \mathbb{R}^{m}$ is the system input, and $y(k) \in \mathbb{R}^{l}$ is the system output. $A \in \mathbb{R}^{n \times n}$, $B \in \mathbb{R}^{n \times n}, g \in \mathbb{R}^{n \times m}$, and $h \in \mathbb{R}^{l \times n}$ are the system parameter matrices and vectors.

Since Equation (1) contains the term $x(k-1)$, we say that this state equation has a unit time-delay. In the following, the state space model with time-delay in Equations (1) and (2) will be transformed into an inputoutput representation and its transfer function will be obtained.

Lemma1. For the state space model in Equations (1) and (2), the transfer function from the input $u_{i}(k)$ $(i=1,2, \ldots, m)$ to the output $y_{j}(k)(j=1,2, \ldots, l)$ is given by

$$
T(q)=h\left(q^{2}-A q-B\right)^{-1} g q
$$

where $q$ represents a unit forward operator that can be shown as

$$
q x(k)=x(k+1) \& q^{-1} x(k)=x(k-1)
$$

Proof: the proof is presented in [16].

Now, for a second-order system with two inputs and two outputs with the following matrices' coefficients:

$$
\begin{aligned}
& A=\left[\begin{array}{ll}
a_{11} & a_{12} \\
a_{21} & a_{22}
\end{array}\right] \in \mathbb{R}^{2 \times 2}, B=\left[\begin{array}{ll}
b_{11} & b_{12} \\
b_{21} & b_{22}
\end{array}\right] \in \mathbb{R}^{2 \times 2} \\
& g=\left[\begin{array}{ll}
g_{11} & g_{12} \\
g_{21} & g_{22}
\end{array}\right] \in \mathbb{R}^{2 \times 2}, h=\left[\begin{array}{ll}
h_{11} & h_{12} \\
h_{21} & h_{22}
\end{array}\right] \in \mathbb{R}^{2 \times 2}
\end{aligned}
$$

Transfer function of the system from the input $u_{1}(k)$ to the output $y_{1}(k)$ will be,

$$
\begin{aligned}
T_{11}(q)= & -g_{21}\left(\frac{h_{11}\left(a_{12} q^{2}+b_{12} q\right)-h_{12}\left(-q^{3}+a_{11} q^{2}+b_{11} q\right)}{d e n}\right) \\
& -g_{11}\left(\frac{h_{12}\left(a_{21} q^{2}+b_{21} q\right)-h_{11}\left(-q^{3}+a_{22} q^{2}+b_{22} q\right)}{\operatorname{den}}\right)
\end{aligned}
$$

where

$$
\begin{aligned}
\operatorname{den}= & \left(b_{12} b_{21}-b_{11} b_{22}\right)+\left(a_{12} b_{21}+a_{21} b_{12}-a_{11} b_{22}-a_{22} b_{11}\right) q \\
& +\left(b_{11}+b_{22}-a_{11} a_{22}+a_{12} a_{21}\right) q^{2}+\left(a_{11}+a_{22}\right) q^{3}-q^{4}
\end{aligned}
$$

The other transfer functions have the same structure as $T_{11}(q)$.

\section{Augmented System}

In this section, using the system dynamic with a unit time-delay, a new state vector called augmented state vector is defined. Therefore an augmented system will be derived from the system with unit time-delay.

The augmented state vector is defined as:

$$
X(k)=\left[\begin{array}{c}
x(k) \\
x(k-1)
\end{array}\right] \Rightarrow X(k+1)=\left[\begin{array}{c}
x(k+1) \\
x(k)
\end{array}\right]
$$

where $x(k)$ is the state vector of the main system and $x(k-1)$ is the state vector with a unit time-delay. Then the system equations are rewritten as

$$
\begin{gathered}
X(k+1)=F X(k)+G u(k) \\
y(k)=H X(k)+v(k)
\end{gathered}
$$

where

$$
\begin{gathered}
F=\left[\begin{array}{ll}
A & B \\
I_{n} & 0
\end{array}\right] \in \mathbb{R}^{2 n \times 2 n}, G=\left[\begin{array}{l}
g \\
0
\end{array}\right] \in \mathbb{R}^{2 n \times m} \\
H=\left[\begin{array}{ll}
h & 0
\end{array}\right] \in \mathbb{R}^{l \times 2 n}
\end{gathered}
$$

Using the properties of the shift operator $q$, the transfer function of the augmented system can be derived from the input $u_{i}(k)$ to the output $y_{j}(k)$ as:

$$
T^{\prime}(q)=H(q I-F)^{-1} G
$$

Now, for a second-order system with two inputs and two outputs with the following matrices' coefficients:

$$
\begin{aligned}
& A=\left[\begin{array}{ll}
a_{11} & a_{12} \\
a_{21} & a_{22}
\end{array}\right] \in \mathbb{R}^{2 \times 2}, B=\left[\begin{array}{ll}
b_{11} & b_{12} \\
b_{21} & b_{22}
\end{array}\right] \in \mathbb{R}^{2 \times 2} \\
& g=\left[\begin{array}{ll}
g_{11} & g_{12} \\
g_{21} & g_{22}
\end{array}\right] \in \mathbb{R}^{2 \times 2}, h=\left[\begin{array}{ll}
h_{11} & h_{12} \\
h_{21} & h_{22}
\end{array}\right] \in \mathbb{R}^{2 \times 2}
\end{aligned}
$$


The augmented system will be a fourth-order system with the following matrices' coefficients:

$F=\left[\begin{array}{cccc}a_{11} & a_{12} & b_{11} & b_{12} \\ a_{21} & a_{22} & b_{21} & b_{22} \\ 1 & 0 & 0 & 0 \\ 0 & 1 & 0 & 0\end{array}\right] \in \mathbb{R}^{4 \times 4}, G=\left[\begin{array}{cc}g_{11} & g_{12} \\ g_{21} & g_{22} \\ 0 & 0 \\ 0 & 0\end{array}\right] \in \mathbb{R}^{4 \times 2}$ $H=\left[\begin{array}{llll}h_{11} & h_{12} & 0 & 0 \\ h_{21} & h_{22} & 0 & 0\end{array}\right] \in \mathbb{R}^{2 \times 4}$

The transfer function from the input $u_{1}(k)$ to the output $y_{1}(k)$ will be

$$
\begin{aligned}
T_{11}^{\prime}(q)= & -g_{21}\left(\frac{h_{11}\left(a_{12} q^{2}+b_{12} q\right)-h_{12}\left(-q^{3}+a_{11} q^{2}+b_{11} q\right)}{d e n}\right) \\
& -g_{11}\left(\frac{h_{12}\left(a_{21} q^{2}+b_{21} q\right)-h_{11}\left(-q^{3}+a_{22} q^{2}+b_{22} q\right)}{d e n}\right)
\end{aligned}
$$

where

$$
\begin{aligned}
\text { den }= & \left(b_{12} b_{21}-b_{11} b_{22}\right)+\left(a_{12} b_{21}+a_{21} b_{12}\right. \\
& \left.-a_{11} b_{22}-a_{22} b_{11}\right) q+\left(b_{11}+b_{22}-a_{11} a_{22}\right. \\
& \left.+a_{12} a_{21}\right) q^{2}+\left(a_{11}+a_{22}\right) q^{3}-q^{4}
\end{aligned}
$$

From Equation (5) and Equation (11) it is clear that $T_{11}(q)=T_{11}^{\prime}(q)$. Therefore, it can be concluded that the system with unit time-delay and the augmented system are equivalent. As a result, by estimating the states of the augmented system, the states of the main system will be estimated. In the next section, the Kalman filter algorithm will be implemented to estimate the states of the augmented system.

\section{Kalman Filter Algorithm for Augmented System}

In order to use the Kalman filter to remove noise from a signal, the process must be described by a linear system. A linear system is simply a process that can be described by Equations (8) and (9). Now, we briefly describe the Kalman filter algorithm to estimate the states as follows:

1. The dynamic system is given by the following equations:

$$
X(k+1)=F X(k)+G u(k)+w(k)
$$

$$
\begin{aligned}
y(k) & =H X(k)+v(k) \\
E\left[w_{k} w_{j}^{T}\right] & =Q_{k} \delta_{k-j} \\
E\left[v_{k} v_{j}^{T}\right] & =R_{k} \delta_{k-j} \\
E\left[v_{k} w_{k}^{T}\right] & =0
\end{aligned}
$$

where $u(k)$ is a known input, $w(k)$ and $v(k)$ are white noise sequences with zero mean and variances $Q_{k}$ and $R_{k}$ respectively. $w(k)$ is called process noise and $v(k)$ is called measurement noise. In practice, the process noise covariance $Q$ and measurement noise covariance $R$ matrices might be time varying, however here we assume that they are constant.

2. The Kalman filter is initialized as follows:

Expected value of the initial state $X_{0}$

$$
\hat{X}_{0}^{+}=E\left(X_{0}\right)
$$

Covariance of initial estimate of $X_{0}$

$$
P_{0}^{+}=E\left[\left(X_{0}-\hat{X}_{0}^{+}\right)\left(X_{0}-\hat{X}_{0}^{+}\right)^{T}\right]
$$

3. The Kalman filter is given by the following equations, which are computed for each step $\mathrm{k}=1,2, \ldots$

$$
\begin{aligned}
& P_{k}^{-}=F P_{k-1}^{+} F^{T}+Q_{k-1} \\
& K_{k}=P_{k}^{-} H^{T}\left(H P_{k}^{-} H^{T}+R\right)^{-1} \\
& \hat{X}_{k}^{-}=F \hat{X}_{k-1}^{+}+G u_{k-1} \\
& \hat{X}_{k}^{+}=\hat{X}_{k}^{-}+K_{k}\left(y_{k}-H \hat{X}_{k}^{-}\right) \\
& P_{k}^{+}=\left(I-K_{k} H\right) P_{k}^{-}\left(I-K_{k} H\right)^{T}+K_{k} R K_{k}^{T}
\end{aligned}
$$

In each time step of the above Kalman filter algorithm, we compute: estimation error covariance of $\hat{X}_{k}^{-}$, Kalman filter gain, a priori estimate of $X_{k}$, a posterior estimate of $X_{k}$, and also the estimation error covariance of $\hat{X}_{k}^{+}$. In the mentioned algorithm, Equations (20) and (22) form time update steps, and Equations (21), (23) and (24) form measurement update steps that are shown in Figure 1.

The next section will be allocated to estimate the states of a second-order system with a unit time-delay by presenting two examples. 


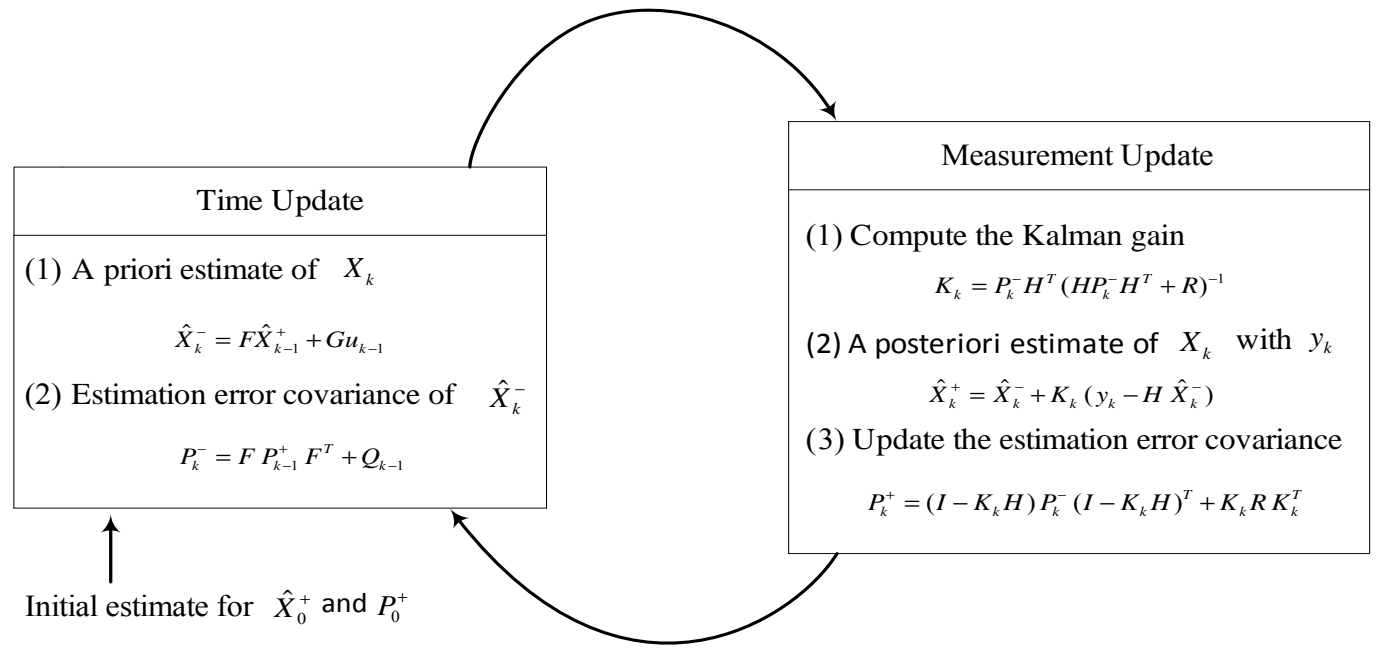

Figure 1. Operation of the Kalman filter, combining Equation (18) to Equation (24).

\section{Simulation Results}

In this Section, we apply the proposed method for both SISO and MIMO systems as follows:

\subsection{SISO System}

Consider the following second-order system

$$
\begin{aligned}
x(k+1) & =\left[\begin{array}{cc}
0 & 1.00 \\
-1.20 & -0.60
\end{array}\right] x(k)+\left[\begin{array}{cc}
0.20 & 0.30 \\
-0.30 & 0.40
\end{array}\right] x(k-1) \\
+ & {\left[\begin{array}{c}
1.00 \\
0.20
\end{array}\right] u(k)+w(k) } \\
y(k) & =\left[\begin{array}{ll}
1.20 & 1.75
\end{array}\right] x(k)+v(k) \\
E\left[w_{k} w_{j}^{T}\right] & =Q_{k} \delta_{k-j}, E\left[v_{k} v_{j}^{T}\right]=R_{k} \delta_{k-j}, E\left[v_{k} w_{k}^{T}\right]=0 \\
Q & =\left[\begin{array}{cc}
\sigma^{2} & 0 \\
0 & \sigma^{2}
\end{array}\right], R=0.25
\end{aligned}
$$

where, the input $u(k) \in \mathbb{R}^{2 \times 1}$ is taken as an uncorrelated persistent excitation signal sequence with zero mean and unit variance, $v(k) \in \mathbb{R}^{2 \times 1}$ and $w(k) \in \mathbb{R}^{2 \times 1}$ are white noise sequences with zero mean and variance $\sigma^{2}$.

Matrices' coefficients of the augmented system will be

$$
\begin{aligned}
F & =\left[\begin{array}{cccc}
0 & 1.00 & 0.20 & 0.30 \\
-1.20 & -0.60 & -0.30 & 0.40 \\
1 & 0 & 0 & 0 \\
0 & 1 & 0 & 0
\end{array}\right], G=\left[\begin{array}{c}
1.00 \\
0.20 \\
0 \\
0
\end{array}\right] \\
H & =\left[\begin{array}{llll}
1.20 & 1.75 & 0 & 0
\end{array}\right]
\end{aligned}
$$

Measurement noise covariance doesn't change but process noise covariance matrix will be changed to

$$
Q=\left[\begin{array}{cccc}
\sigma^{2} & 0 & 0 & 0 \\
0 & \sigma^{2} & 0 & 0 \\
0 & 0 & \sigma^{2} & 0 \\
0 & 0 & 0 & \sigma^{2}
\end{array}\right]
$$

With the following initial state vector and estimation error covariance matrices'

$$
\hat{X}_{0}^{+}=\left[\begin{array}{l}
3 \\
2 \\
6 \\
3
\end{array}\right], P_{0}^{+}=\left[\begin{array}{cccc}
100 & 0 & 0 & 0 \\
0 & 10 & 0 & 0 \\
0 & 0 & 10 & 0 \\
0 & 0 & 0 & 1
\end{array}\right]
$$

We run the simulation for $\sigma^{2}=0$ and also $\sigma^{2}=0.5^{2}$.

It is clear from Figures 2 and 3 that decreasing in process noise covariance $\left(\sigma^{2}\right)$ causes more similarity in the estimated states and the true states. With regard to this subject that the augmented system is used for estimating the states, for comparison between estimated states and true states, only the first and the second rows of the augmented state vector, that are the main states (without time-delay), will be used.

On the other hand, we can see unit time-delay in the state vector as follows:

At each time step, the third and fourth rows of the augmented state vector that are with a unit time-delay, are very close to the first and the second rows of the augmented state vector at previous time step. And if we 

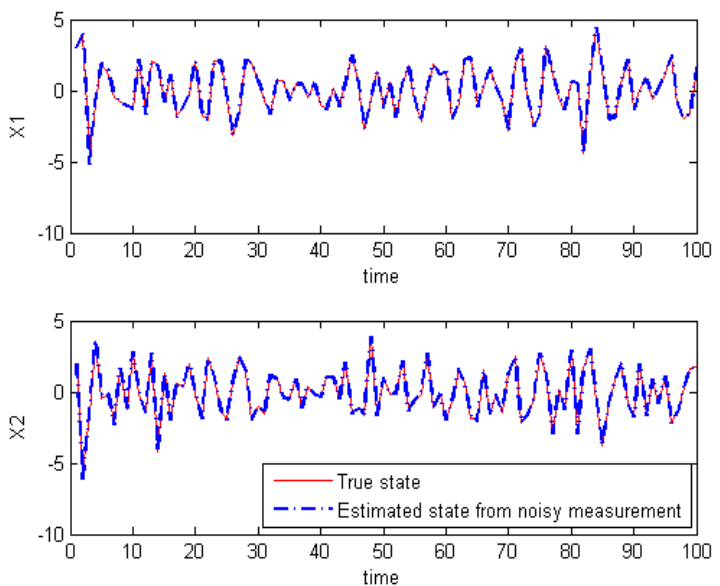

Figure 2. Estimated states and true states $\left(\sigma^{2}=0\right)$.
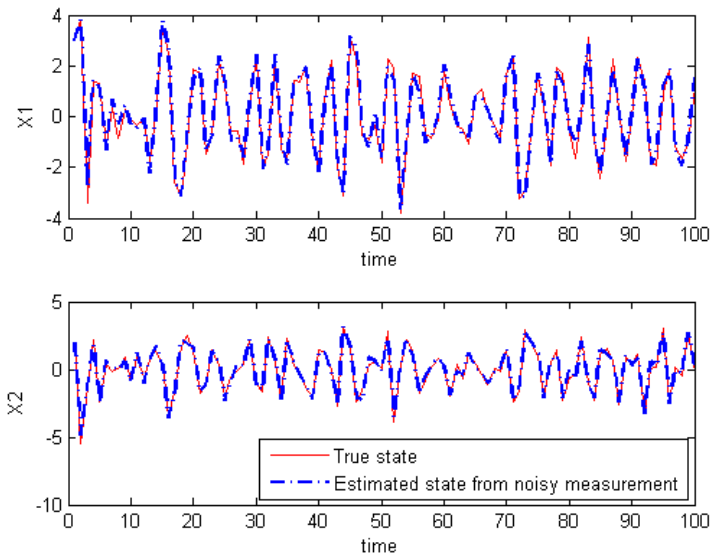

Figure 3. Estimated states and true states $\left(\sigma^{2}=0.25\right)$.

don't have process noise in the Kalman filter algorithm $\left(\sigma^{2}=0\right)$, then in $k^{\text {th }}$ time step of the third and the fourth rows of the augmented state vector will be equal to the first and the second rows at (k-1) th time step. For example, the augmented state vector for $\sigma^{2}=0$ and $\sigma^{2}=0.5^{2}$ in 10 times step is presented in Table 1.
In Figures 4 and 5, it can be seen that the estimation error covariance decrease over time steps and finally will be in a good region. For $\sigma^{2}=0$ it will be equal to:

$$
P=1.0 e-035 \times\left[\begin{array}{rrrr}
0.0684 & -0.0011 & 0.0017 & 0.0766 \\
-0.0011 & 0.0602 & -0.0801 & 0.0294 \\
0.0017 & -0.1066 & 0.1066 & -0.0388 \\
0.0766 & 0.0294 & -0.0388 & 0.1012
\end{array}\right]
$$

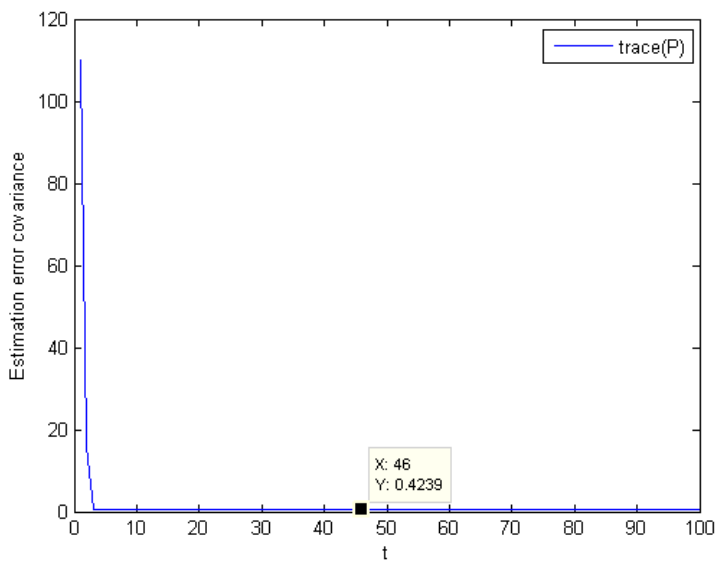

Figure 4. Trace of estimation error covariance matrix $\left(\sigma^{2}=0\right)$.

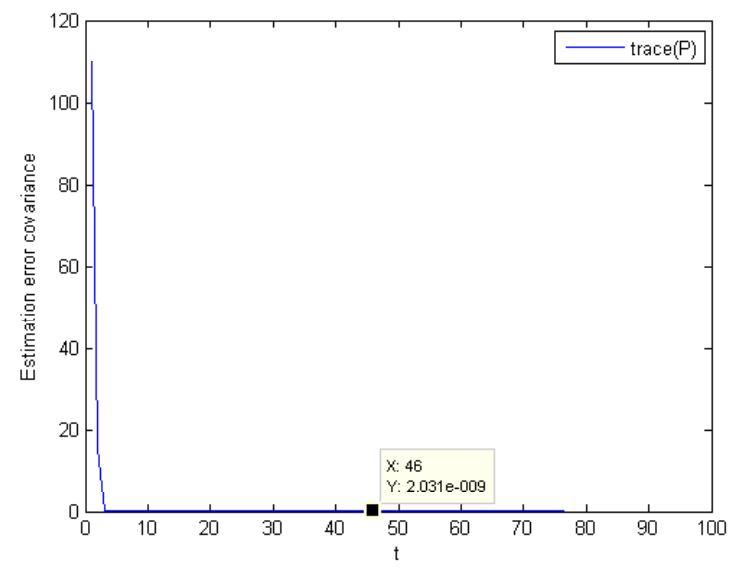

Figure 5. Trace of estimation error covariance matrix $\left(\sigma^{2}=0\right)$.

Table 1. Augmented state vector in 10 time steps (from $\mathrm{k}=20$ to $\mathrm{k}=30$ ), (a): $\sigma^{2}=0$, (b) : $\sigma^{2}=0.5^{2}$

\begin{tabular}{|c|c|c|c|c|c|c|c|c|c|c|}
\hline$X 1$ & -0.817 & -0.373 & 0.048 & 1.634 & 0.948 & -0.823 & -0.243 & 0.936 & -0.088 & -1.587 \\
\hline $\mathrm{X} 2$ & 1.312 & 0.366 & 0.888 & -0.190 & -1.334 & -0.855 & 0.848 & -0.210 & -0.601 & -0.120 \\
\hline X3 & -1.665 & -0.817 & -0.373 & 0.048 & 1.634 & 0.948 & -0.823 & -0.243 & 0.936 & -0.088 \\
\hline$X 4$ & -0.166 & 1.312 & 0.366 & 0.888 & $\begin{array}{r}-0.190 \\
\text { (a) }\end{array}$ & -1.334 & -0.855 & 0.848 & -0.210 & -0.601 \\
\hline $\mathrm{X} 1$ & -2.468 & -3.114 & -0.852 & 1.489 & 1.914 & -1.379 & -0.698 & 2.358 & 1.325 & -0.941 \\
\hline $\mathrm{X} 2$ & -1.955 & 2.134 & 1.912 & 1.573 & -1.632 & -1.212 & 1.351 & 0.548 & -2.296 & -0.800 \\
\hline $\mathrm{X} 3$ & 2.6560 & -2.695 & -2.986 & -0.890 & 1.450 & 1.911 & -1.418 & -0.833 & 2.395 & 1.452 \\
\hline$X 4$ & -3.536 & -1.838 & 2.056 & 1.935 & $\begin{array}{r}1.597 \\
\text { (b) }\end{array}$ & -1.631 & -1.188 & 1.439 & 0.526 & -2.373 \\
\hline
\end{tabular}


and for $\sigma^{2}=0.5^{2}$ it will be:

$$
P=\left[\begin{array}{rrrr}
0.2685 & -0.1511 & -0.0161 & 0.0711 \\
-0.1511 & 0.1554 & -0.0146 & -0.0331 \\
-0.0161 & -0.0146 & 0.4276 & -0.0955 \\
0.0711 & -0.0331 & -0.0955 & 0.3714
\end{array}\right]
$$

\subsection{MIMO System}

The simulation results for a second order system with two inputs and two outputs with the following equations is shown in this sub-section,

$$
\begin{aligned}
x(k+1)= & {\left[\begin{array}{ll}
0.20 & 0.20 \\
0.10 & 0.40
\end{array}\right] x(k)+\left[\begin{array}{cc}
0.50 & 0.20 \\
-0.20 & -0.10
\end{array}\right] x(k-1) } \\
& +\left[\begin{array}{ll}
2 & 1 \\
1 & 2
\end{array}\right] u(k)+w(k) \\
y(k)= & {\left[\begin{array}{ll}
1 & 0 \\
0 & 1
\end{array}\right] x(k)+v(k) } \\
E\left[w_{k} w_{j}^{T}\right]= & Q_{k} \delta_{k-j}, E\left[v_{k} v_{j}^{T}\right]=R_{k} \delta_{k-j}, E\left[v_{k} w_{k}^{T}\right]=0 \\
Q= & {\left[\begin{array}{cc}
\sigma^{2} & 0 \\
0 & \sigma^{2}
\end{array}\right], R=\left[\begin{array}{cc}
0.25 & 0 \\
0 & 0.25
\end{array}\right] }
\end{aligned}
$$

where, the input $u(k) \in \mathrm{i}^{2 \times 1}$ is taken as an uncorrelated persistent excitation signal sequence with zero mean and unit variance, $v(k) \in \mathrm{i}^{2 \times 1}$ and $w(k) \in \mathrm{i}^{2 \times 1}$ are white noise sequences with zero mean and variance $\sigma^{2}$.

Matrices' coefficients of the augmented system will be

$$
\begin{aligned}
F & =\left[\begin{array}{cccc}
0.2 & 0.2 & 0.50 & 0.20 \\
0.10 & 0.40 & -0.20 & -0.10 \\
1 & 0 & 0 & 0 \\
0 & 1 & 0 & 0
\end{array}\right], G=\left[\begin{array}{ll}
2 & 1 \\
1 & 2 \\
0 & 0 \\
0 & 0
\end{array}\right] \\
H & =\left[\begin{array}{llll}
1 & 0 & 0 & 0 \\
0 & 1 & 0 & 0
\end{array}\right]
\end{aligned}
$$

Now with the following initial state vector and estimation error covariance matrices'

$$
\hat{X}_{0}^{+}=\left[\begin{array}{l}
3 \\
2 \\
6 \\
3
\end{array}\right], P_{0}^{+}=\left[\begin{array}{cccc}
100 & 0 & 0 & 0 \\
0 & 10 & 0 & 0 \\
0 & 0 & 10 & 0 \\
0 & 0 & 0 & 1
\end{array}\right]
$$

run the simulation for $\sigma^{2}=0$ and also $\sigma^{2}=0.5^{2}$.
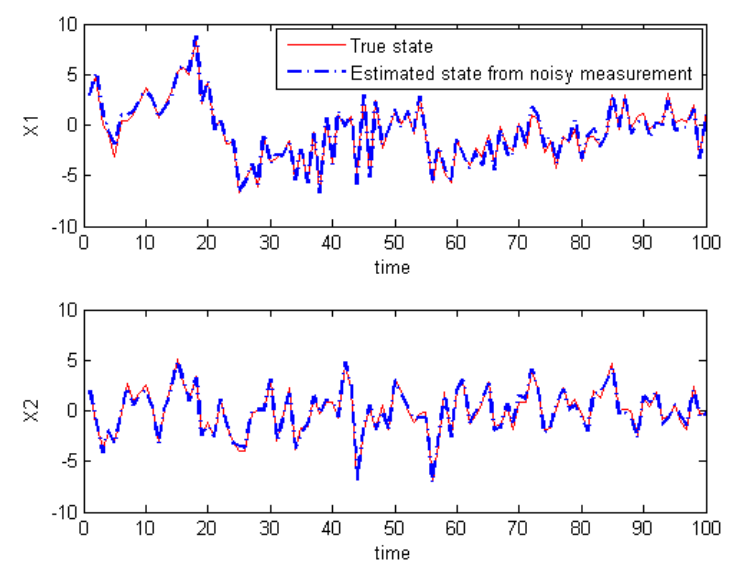

Figure 6. Estimated states and true states $\left(\sigma^{2}=0\right)$.
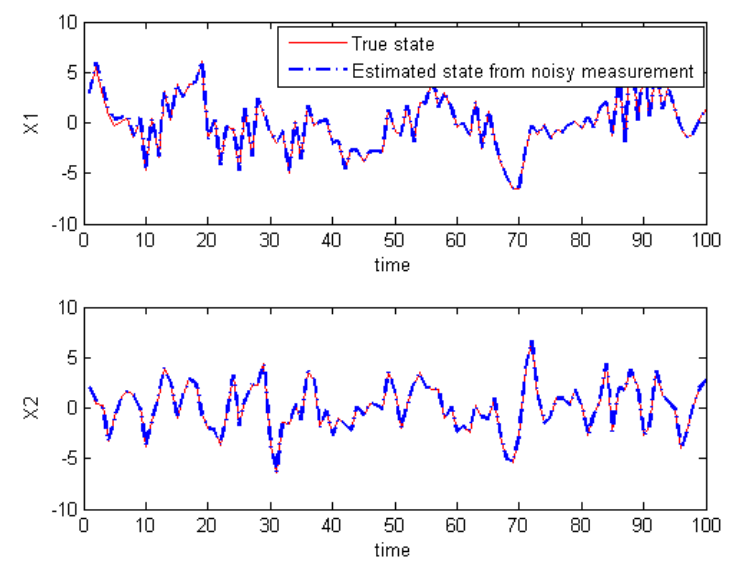

Figure 7. Estimated states and true states $\left(\sigma^{2}=0.5^{2}\right)$.

From Figures 6 and 7 it can be seen that decreasing in process noise covariance $\left(\sigma^{2}\right)$ causes more similarity in the estimated states and the true states and from Figure 8 and 9 it is clear that the estimation error covariance decrease over time steps and finally will be in a good region as well as SISO system.

For $\sigma^{2}=0$ it will be equal to:

$$
P=1.0 e-059 \times\left[\begin{array}{rrrr}
0.3362 & -0.1354 & 0.4735 & -0.1907 \\
-0.1354 & 0.0546 & -0.1907 & 0.0768 \\
0.4735 & -0.1907 & 0.6668 & -0.2686 \\
-0.1907 & 0.0768 & -0.2686 & 0.1082
\end{array}\right]
$$

and for $\sigma^{2}=0.5^{2}$ it will be:

$$
P=\left[\begin{array}{rrrr}
0.1507 & -0.0057 & 0.0160 & 0.0141 \\
-0.0057 & 0.1335 & 0.0047 & 0.0238 \\
0.0160 & 0.0047 & 0.3979 & -0.0088 \\
0.0141 & 0.0238 & -0.0088 & 0.3770
\end{array}\right]
$$




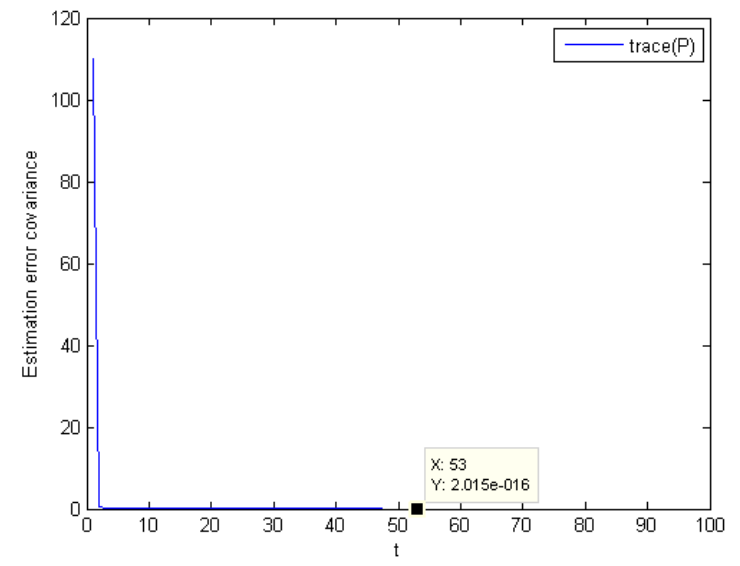

Figure 8. Trace of estimation error covariance matrix $\left(\sigma^{2}=0\right)$.

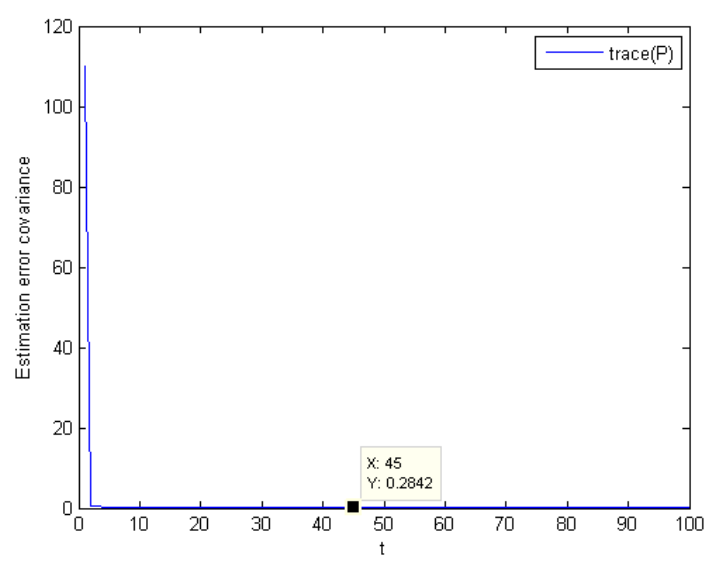

Figure 9. Trace of estimation error covariance matrix $\left(\sigma^{2}=0.5^{2}\right)$.

\section{Conclusion}

This paper discusses state estimation problem for a multi-input multi-output linear system with unit timedelay using Kalman filter. An augmented system was presented for estimating the states. Required matrices for Kalman filter algorithm in a second-order system were calculated and it was shown that the main system with unit time-delay and the augmented system are equivalent. Finally, performance of the proposed algorithm was studied using an illustrative example in which the estimation error covariance had an acceptable value.

\section{References}

1. Mercère G, and Bako L (2011). Parameterization and identification of multivariable state-space systems:
A canonical approach, Automatica, vol 47(8), $1547-1555$.

2. Xu X, Shi Z Y et al. (2012). Identification of linear time-varying systems using a wavelet-based state-space method, Mechanical Systems and Signal Processing, vol 26, 91-103.

3. Kou P, Zhou J et al. (2011).Parameters identification of nonlinear state space model of synchronous generator, Engineering Applications of Artificial Intelligence, vol 24(7), 1227-1237.

4. Subrahmanya N, and Shin Y C (2009). Adaptive divided difference filtering for simultaneous state and parameter estimation, Automatica, vol 45(7), 1686-1693.

5. Junping W, and Jingang G (2009), An adaptive Kalman filtering based State of Charge combined estimator for electric vehicle battery pack, Energy Conversion and Management, vol 50(12), 3182-3186.

6. Shi Y, and Fang H Z (2010). Kalman filter-based identification for systems with randomly missing measurements in a network environment, International Journal of Control, vol 83(3), 538-551.

7. Zhang J B, and Ding F et al. (2009). Self-tuning control based on multi-innovation stochastic gradient parameter estimation, Systems \& Control Letters, vol 58(1), 69-75.

8. Sun J, Zuo H et al. (2012). Application of a state space modeling technique to system prognostics based on a health index for condition-based maintenance, Mechanical Systems and Signal Processing, vol 28, 585-596.

9. Miranda H, Cortes P et al. (2009). Predictive Torque Control of Induction Machines Based on State-Space Models, IEEE Transactions on Industrial Electronics, vol 56(6), 1916-1924.

10. Dehghani M, and Nikravesh S (2008). State-Space Model Parameter Identification in Large-Scale Power Systems, IEEE Transactions on Power Systems, vol 23(3), 1449-1457.

11. Thalmayr F, and Fischerauer G (2010). Sensor signal processing for gravimetric chemical sensors based on a state-space model, Sensors and Actuators B: Chemical, vol 144(1), 27-36.

12. Zheng Y R, Shao T et al. (2012). A fast-converging spacetime adaptive processing algorithm for non-Gaussian clutter suppression, Digital Signal Processing, vol 22(1), 74-86.

13. Yan M, and Shi Y (2008). Robust discrete-time sliding mode control for uncertain systems with time-varying state delay, IET Control Theory \& Applications, vol 2(8), 662-674.

14. Shi Y, and Yu B (2009). Output feedback stabilization of networked control systems with random delays 
modeled by Markov chains, IEEE Transactions on Automatic Control, vol 54(7), 1668-1674.

15. Shi L, Xie L et al. (2009).Kalman filtering over a packetdelaying network: a probabilistic approach, Automatica, vol 45(9), 2134-2140.
16. Gu Y, and Ding F (2012). Auxiliary model based least squares identification method for a state space model with a unit time-delay, Applied Mathematical Modelling, vol 36(12), 5773-5779. 